\title{
Dosimetric comparison of radiation methods for palmar fibrosis
}

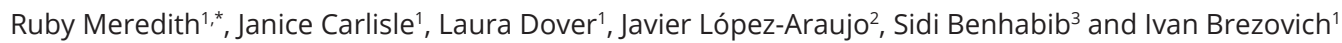

\author{
'Department of Radiation Oncology, University of Alabama at Birmingham, Birmingham, AL, USA \\ ${ }^{2}$ Auxilio Mutuo Radiation Oncology Center, Ponce de Leon 725 Pda. 37.5, Hato Rey, P.R., 00917, USA \\ ${ }^{3}$ Inova Dwight and Martha Schar Cancer Institute, 3300 Gallows Road, Falls Church VA 22042, USA
}

\begin{abstract}
Purpose: Dosimetric comparison was performed of radiation methods for target coverage and non-target sparing in palmar fibrosis (i.e., Dupuytren disease). Most early stage disease has been treated with superficial radiation using standard cones and shielding of uninvolved areas. With clinical set up, no information regarding depth of the nodules or percent coverage of the target was obtained. With concern for late effects of radiation used to treat a benign condition and declining availability of superficial radiation equipment, we compared dosimetry of superficial radiation versus patient specific electron therapy. Methods: One female patient (thinner hand) and one male patient were selected for intra-patient range in a CT dosimetric comparison of 6-MeV electrons \pm bolus versus $90 \mathrm{kV}$ photons. The clinically set up target volume (CTV) included cords/nodules plus 1-2 cm margin. The posterior hand avoidance was defined as the tissues posterior to the ventral border of the metacarpal bones. Results: Normalized to $90 \%$ of the prescribed dose, $6-\mathrm{MeV}$ electrons \pm bolus achieved $100 \%$ coverage of CTV. The dose maximum as well as dose to the posterior hand avoidance was reduced by bolus. The $90 \%$ isodose line with the superficial radiotherapy was $0.4 \mathrm{~cm}$ depth and achieved $73-75 \%$ CTV coverage. Conclusions: 6-MeV electrons with individualized bolus gave superior CTV coverage over superficial radiotherapy at the $90 \%$ isodose lines, with relative sparing of normal tissues.
\end{abstract}

Keywords: palmar fibrosis; Dupuytren's contracture; radiation

\section{Introduction}

Dupuytren disease is a connective tissue proliferative process of the palms with development of nodules and fibrotic cords leading to contracture. Radiation has been moderately effective for halting the process in early stages although it does not reverse contracture. Traditionally, early stage Dupuytren disease was treated with superficial (20-150kV) or orthovoltage (150-500 kV) radiotherapy using standard cones and shielding of uninvolved areas, as was used in the only prospective randomized trial to date [1]. With clinical set up of superficial radiation, no information regarding depth of the nodules or percent coverage of the target is obtained. With concern for late effects of radiation used to treat a benign condition and declining availability of superficial radiation equipment, we compared dosimetry of superficial versus patient specific electron therapy. We also assessed treatment effect and post-treatment toxicity using a patient survey.

\section{Methods}

This study was approved by the University of Alabama Institutional Review Board. Eight of ten patients treated at our institution from 2010-2016 for palmar fibrosis received 30 Gy in 10 fractions using electrons in a split-course regimen similar to that reported in a randomized trial [1].
Treatment consisted of five consecutive days of single $3 \mathrm{~Gy}$ fractions, with a repeat after 8-12 weeks. Typically, the setup included positioning of the hand on a custom molded immobilization device (Alpha Cradle ${ }^{\circledR}$ ), in an open palm, finger extended position (Figure 1). A detailed mapping of the nodules/cords was done clinically and radio-opaque markers placed over the delineated target. Margins of 2 $\mathrm{cm}$ superior/inferior and $1 \mathrm{~cm}$ medial/lateral were applied to the clinical field. CT simulation was obtained for nodule depth using $1 \mathrm{~mm}$ slice thickness. The target was any nodules/cords palpated or defined by CT images. A clinical target volume (CTV) used the clinically placed margins and CT scan depth information plus $2 \mathrm{~mm}$ margin below

\footnotetext{
*Corresponding author: Ruby F. Meredith, MD, PhD, Hazelrig Salter Radiation Oncology Center, 1700 6th Avenue South, Birmingham, AL 35233, USA. Tel.: (205) 975-0224; Fax: (205) 975-0784; Email: rmeredith@uabmc.edu

Received 03 August 2017 Revised 10 October 2017 Accepted 25 October 2017 Published 1 November 2017

Citation: Meredith R, Carlisle J, Dover L, López-Araujo J, Benhabib S, Brezovich I. Dosimetric comparison of radiation methods for palmar fibrosis. J Clin Radiat Oncol. 2017; 2(1):1-3. DOI: 10.14312/2397-8511.2017-1

Copyright: (c) 2017 Meredith R, et al. Published by NobleResearch Publishers. This is an open-access article distributed under the terms of the Creative Commons Attribution License, which permits unrestricted use, distribution and reproduction in any medium, provided the original author and source are credited.
} 
disease and extension to the palmar skin above the target. CTV depth was measured as the perpendicular distance from the palmar skin to the deepest portion of the target. Hand thickness was measured at the epicenter of the CTV. The CTV was contoured in the Varian Eclipse ${ }^{\mathrm{TM}}$ Treatment Planning System; then electron beam energy was chosen to provide adequate coverage of the CTV. Custom tissue equivalent bolus was placed on the palm with dosimetric goals of covering $100 \%$ of the CTV by the $\geq 90 \%$ isodose line. A $10 \times 10 \mathrm{~cm}$ cone was used with an $8 \mathrm{~cm}$ custom cutout. The posterior hand avoidance (PHA) was defined as the tissues posterior to the ventral aspect of the metacarpal bones since all nodules and cords were felt to be superficial to that border.

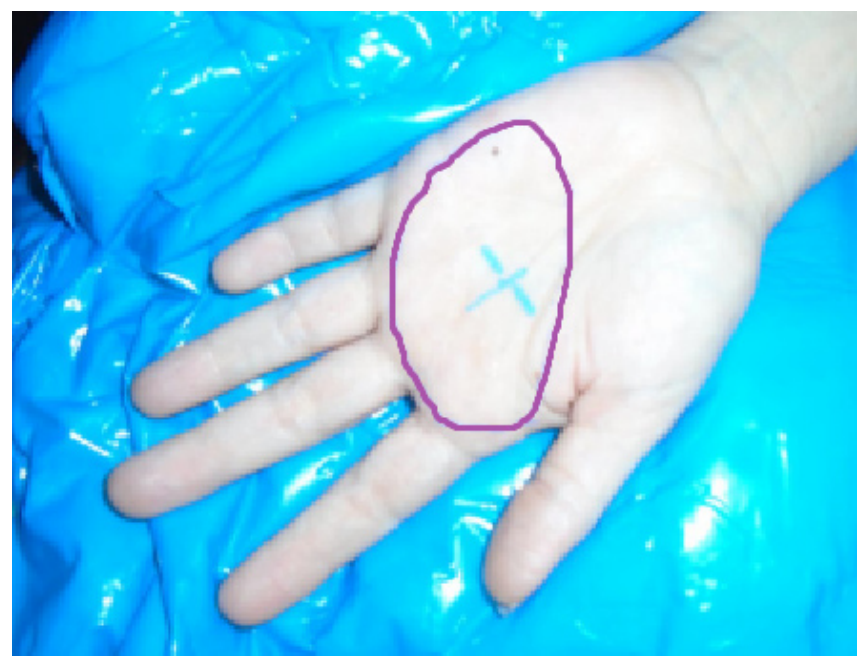

Figure 1 Hand set up in custom molded device.

To assess potential benefits of electrons over traditional superficial radiation techniques, dosimetric analyses were performed comparing 6-MeV electrons \pm bolus versus photons from a superficial unit. One female (Patient 1 ) and one male (Patient 2) were selected for dosimetric intrapatient treatment modality comparisons. 3D-electron calculations were done for 6-MeV electrons using the Eclipse electron macro Monte Carlo algorithm (eMC) with $8 \mathrm{~cm}$ cutout \pm bolus of $5 \mathrm{~mm}$ or $7 \mathrm{~mm}$.

The statistical uncertainty for the dose calculation was fixed to $1 \%$ and grid size of $1 \mathrm{~mm}$. Calculations were also done for our clinical superficial unit (PROFEXRAY serial \# 50755) providing $90 \mathrm{kVp}$ x-rays with $1 \mathrm{~mm}$ Al added filtration, having a half-value-layer of $2.0 \mathrm{~mm} \mathrm{Al}$. A circular cone of $8 \mathrm{~cm}$ was used at a source to surface distance of $15 \mathrm{~cm}$. The calculated dosimetry percents were rounded to the nearest whole number.

\section{Results}

For all hands treated, 6e- was adequate for depth coverage of nodules that were clinically $0.4-0.9 \mathrm{~cm}$. For intra-patient as well as radiation methodology dosimetry comparison, we selected a female patient (Patient 1) as a thinner hand and nodules versus a male patient (Patient 2 ) with a thicker hand and larger nodules.

For Patient 1, hand thickness was $2.68 \mathrm{~cm}$ and CTV was $1.07 \mathrm{~cm}^{3}$ with a depth of $0.94 \mathrm{~cm}$. For Patient 2, hand thickness was $3.19 \mathrm{~cm}$ and CTV was $1.78 \mathrm{~cm}^{3}$ with a depth of $1.1 \mathrm{~cm}$. As seen in Figure 2, for normalization at $90 \%$ of the prescribed dose, $90 \%$ of the dose (D90) achieved $100 \%$ coverage of CTV. This held for both patients using 6-MeV electrons \pm bolus. With bolus, the hot spots were 127$128 \%$ compared to $142-164 \%$ without bolus, and $50 \%$ dose area (D50) of posterior hand avoidance was $11-31 \%$ versus $41-76 \%$, respectively (Table 1 ). Figure 2 demonstrates that with bolus the hot spot is inside the CTV whereas without bolus it is deeper into tissue such that the highest dose is in the avoidance structure. Also, with backscatter from high Z material of bone, the hot spot was up to $164 \%$ (Table 1). The transverse views in Figure 3 illustrate the superior avoidance of normal tissue and more desirable location of hot spots as an effect of bolus utilization. A color wash of dose variation shows that the $90 \%$ isodose extends midway through most of the metacarpals without bolus in patient 1 whereas the addition of bolus excludes the majority of bone and other avoidance tissues.

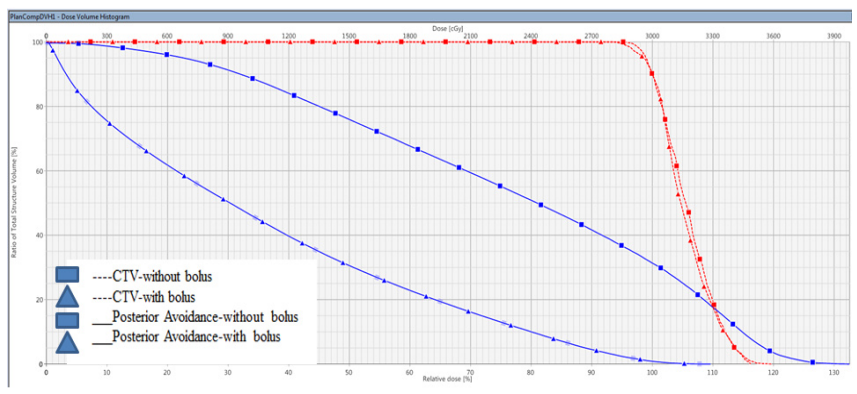

Figure 2 Dose-Volume Histograms of 6e- plans are compared for CTV coverage (- - - dashed lines), and PHA (__ solid lines) without and with 7 $\mathrm{mm}$ bolus for Patient 1 .

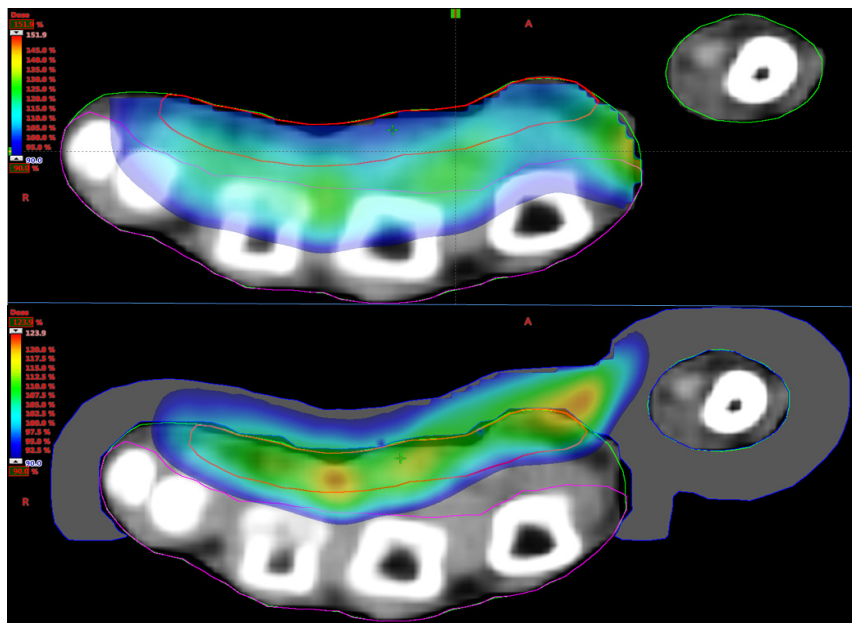

Figure 3 Electron dose distributions for Patient 1 without bolus (upper image), and with $7 \mathrm{~mm}$ bolus (lower image). The red line shows the CTV and the magenta line delineates the avoidance; the inferior edge of the color wash shows $90 \%$ of the prescribed dose.

Table 1 Effect of bolus on the percent maximum dose from electrons and quantity of avoidance receiving half of the prescribed Gy.

\begin{tabular}{lccc}
\hline & No bolus & $5 \mathrm{~mm}$ bolus & $7 \mathrm{~mm}$ bolus \\
\hline Patient 1 & & & \\
\% of Avoidance $\geq$ D50 & 76 & 43 & 31 \\
Maximum dose & 142 & 127 & 127 \\
Patient 2 & & & \\
\% of Avoidance $\geq$ D50 & 41 & 17 & 11 \\
Maximum dose & 164 & 128 & 128 \\
\hline
\end{tabular}


The $90 \%$ isodose line from the superficial radiotherapy was $0.4 \mathrm{~cm}$ depth and achieved $73-75 \%$ CTV coverage. The highest dose, being at the surface, is delivered to keratinized skin overlying the target nodules.

\section{Discussion}

This dosimetry comparison showed the advantage of 6e- over $90 \mathrm{kVp}$ superficial radiation for superior target coverage. It illustrated the beneficial effect of bolus with 6e- therapy which not only kept the dose to avoidance lower, but also placed the hot spot in the CTV rather than in the non-target tissue. Our results indicate that most patients will be adequately treated with $6 \mathrm{e}-$ and $>5 \mathrm{~mm}$ bolus. Although we compared hands of different thickness, the depth of the palmar superficial aponeurosis/nodules, not the hand thickness, is important in choosing depth of bolus. The thickest nodule in our patient cohort was $<1 \mathrm{~cm}$ and most will have $1 \mathrm{~mm}$ or more of skin over the nodules; thus coverage at the most palmar aspect of the nodules would be $>85 \%$. With this set up, coverage at 2 $\mathrm{cm}$ is $76 \%$, which provides dose as good as superficial radiation, should nodules be of such thickness to require $2 \mathrm{~cm}$ of depth coverage. For patients with small nodules, 7 $\mathrm{mm}$ bolus is preferred to provide $90 \%$ dose at the ventral side of nodules and adequate penetration for the deepest portion. This study directly compared 6e- with $90 \mathrm{kVp}$ since that was the superficial radiation available at our center. Many Radiation Oncology centers no longer maintain superficial radiation equipment but should be able to offer 6e- therapy.

Despite clinically placed markers and CT slices of only 1 $\mathrm{mm}$ in our patients, palpable nodules were not always discernable on images. Thus, considering the CT limitations and results of our dosimetry comparison, clinical set up is adequate. Since the fibrosis process generally does not extend to the metacarpals, our defined CTV should cover the target tissue. Clinical set up with or without custom molded immobilization may allow the patients to be treated with hands at sides, whereas CT set up used arms overhead to contain CT to the hands. Arms at sides is more comfortable than overhead. However, a linear accelerator table may require a lateral extension to be wide enough for treatment of outstretched hands at sides. Custom molded devices especially facilitated set up for the second portion of treatment as initial field marks were no longer visible.

Betz et al. report favorable outcome for radiation of early stage Dupuytren disease after 13 years [2-6]. Late effects of erythema or stiffness have been noted after superficial radiation in prior publications but no reports of malignancy induction were found $[2,4]$. Our margins of $1-2 \mathrm{~cm}$ were larger than 2015 DEGRO guidelines but were based on methods used in the previous randomized trial and are similar to margins used in patients for whom longer followup is reported $[1,4,7]$.

\section{Conclusions}

Based on numerous reports, radiation for early stage palmar fibrosis is safe and effective for the majority of patients. 1) Use of 6e- with bolus provides superior target coverage over $90 \mathrm{kVp}$ superficial photons. Bolus of $>5 \mathrm{~mm}$ is advantageous for small nodules. 2) Clinical set up is adequate and simpler than CT planning.

\section{Conflicts of interest}

Authors declare no conflicts of interest.

\section{References}

[1] Seegenschmiedt MH, Olschewsk T, Guntrum F. Radiotherapy optimization in early-stage Dupuytren's contracture: first results of a randomized clinical study. Int J Radiat Oncol Biol Phys. 2001; 49(3):785798.

[2] Eberlein B, Biedermann T. To remember: Radiotherapy - a successful treatment for early Dupuytren's disease.J Eur Acad Dermatol Venereol. 2016; 30(10):1694-1699.

[3] Schuster J, Saraiya S, Tennyson N, Nedelka M, Mukhopadhyay N, et al. Patient-reported outcomes after electron radiation treatment for early-stage palmar and plantar fibromatosis. Pract Radiat Oncol. 2015; 5(6):e651-658.

[4] Betz N, Ott O, Adamietz B, Sauer R, Fietkau R, et al. Radiotherapy in early-stage Dupuytren's contracture. Long-term results after 13 years. Strahlenther Onkol. 2010; 186(2):82-90.

[5] Zirbs M, Anzeneder T, Bruckbauer B, Hofmann H, Brockow K, et al. Radiotherapy with soft X-rays in Dupuytren's disease - successful, well-tolerated and satisfying. J Eur Acad Dermatol Venereol. 2015; 29(5):904-911.

[6] McKeown SR, Hatfield P, Prestwich RJ, Shaffer RE, Taylor RE. Radiotherapy for benign disease; assessing the risk of radiationinduced cancer following exposure to intermediate dose radiation. $\mathrm{Br}$ J Rad. 2015; 88(1056): 20150405.

[7] Seegenschmiedt MH, Micke O, Niewald M, Mucke R. DEGRO guidelines for the radiotherapy of non-malignant disorders: Part III: Hyperproliferative disorders. Strahlenther Onkol. 2015. 191(7):541548. 